3. Zammarchi, F., et al., A CD25-targeted pyrrolobenzodiazepine dimer-based antibody-drug conjugate shows potent anti-tumor activity in pre-clinical models of solid tumors either alone or in combination with a PD-1 inhibitor. Journal for ImmunoTherapy of Cancer 2018. 6(Supplement 1).

4. Zammarchi, F., et al., A CD25-targeted antibody-drug conjugate depletes regulatory $T$ cells and eliminates established syngeneic tumors via antitumor immunity. Journal for Immunotherapy of Cancer 2020. In press.

http://dx.doi.org/10.1136/jitc-2020-SITC2020.0621

\section{PD-L1 IS A POTENTIAL PREDICTIVE BIOMARKER FOR RESPONSE TO RM-1929 TREATMENT IN RECURRENT HEAD AND NECK SQUAMOUS CELL CARCINOMA PATIENTS}

C Daniel De Magalhaes Filho*, Chung-Wein Lee, Nikolai Suslov, Jerry Fong, Miguel GarciaGuzman. Rakuten Medical, San Diego, CA, USA

Background RM-1929 is an antibody-dye conjugate comprised of cetuximab covalently linked to the photoactivable dye, IRDye $^{\circledR}$ 700DX (IR700). After systemic infusion of RM-1929, illumination of the tumor with $690 \mathrm{~nm}$ non-thermal red light activates the drug and results in targeted and rapid tumor necrosis. Previous preclinical data have shown that RM-1929 treatment triggers immunogenic cell death and activates the innate and adaptive immune response. A retrospective analysis of PD-L1 expression from the phase I/IIa clinical trial in patients with recurrent head and neck squamous cell carcinoma (rHNSCC) (NCT02422979) was conducted. The analysis explored correlations of PD-L1 expression, including combined proportion score (CPS) and tumor proportion score (TPS), with clinical outcomes such as response rate and overall survival.

Methods PD-L1 expression prior to RM-1929 treatment was assessed by immunohistochemistry in 18 out of 30 patients enrolled in Part II of the trial, based on sample availability. PD-L1 expression was evaluated using TPS and CPS. Responders were defined as patients that achieved complete response or partial response, and non-responders had either stable disease or progressive disease. Overall survival (OS) was analyzed using the Kaplan-Meier method.

Results Responders $(n=10)$ had a TPS of $4.3 \pm 2.4$ (mean \pm SEM), which was substantially lower than in non-responders $(n=8)$ with a TPS of $39.4 \pm 11.8$. Similarly, CPS was lower in responders $(8.6 \pm 3.6)$ compared to non-responders $(50.0$ \pm 13.5 ). The best target response rate for all patients included in this analysis was 56\%. Patients with CPS $=40$ had a response rate of $76.9 \%(n=13)$ compared to $0 \%$ in patients with CPS $>40 \quad(n=5)$. This suggests that a CPS cut-off of $=40$ led to enrichment of the best target response rate. The median OS of patients with CPS $=40(13.0 \pm 0.8$ months $)$ was also higher than in patients with CPS $>40$ (3.1 \pm 0.8 months) and in all patients $(12.0 \pm 2.9$ months).

Conclusions These results suggest that rHNSCC patients with lower PD-L1 expression levels may be more responsive to RM-1929 treatment and CPS/TPS could potentially be predictive biomarkers in identifying patients with a higher probability of benefiting from this treatment. Given the limited number of patients in this analysis, additional clinical trials will be needed to validate PD-L1 expression as an effective predictive biomarker for RM-1929 treatment.

Acknowledgements The authors would like to thank all patients and their families for their participation in this trial. The authors would also like to thank the following investigators for the contribution of samples included in this trial analysis: Dr. David Cognetti (Thomas Jefferson University Hospital), Dr. Ann M Gillenwater (University of Texas MD Anderson Cancer Center), Dr. Mary Jo Fidler (Rush University Medical Center), Dr. Samith T. Kochuparambil (Virginia Piper Cancer Institute ), Dr. John Campana (University of Colorado Head and Neck Specialists), and Dr. Nilesh R. Vasan (University of Oklahoma Health Sciences Center).

Trial Registration NCT02422979

Ethics Approval The trial was approved by the following Instution Ethics Boards and IRB\# as listed: UCSF Institutional Review Board (\#17-21904), Thomas Jefferson University, IRB (\#16C.328), University of Oklahoma Health Sciences Center Institutional Review Board (\#5723), University of Texas MD Anderson Cancer Center - Institutional Review Board (\#IRB 2 IRB00002203), Quorum Review IRB (\#30458/1), Rush University Medical Center Institutional Review Board (\#15030601-IRB01), and Catholic Health Initiatives Institute for Research and Innovation (CIRI) Institutional Review Board (CHIRB) (\# IRB00009715).

Consent N/A

http://dx.doi.org/10.1136/jitc-2020-SITC2020.0622

\section{IMMUNO-STATS: LEVERAGING PROTEIN ENGINEERING TO EXPAND AND TRACK ANTIGEN-SPECIFIC T CELLS IN VIVO}

${ }^{1}$ Stad Zeigler, ${ }^{2}$ Andrew Woodham, 'Mengyan Li, ${ }^{3}$ Ella Zeyang, ${ }^{2}$ Stephen Kolifrath, ${ }^{4}$ Mohammad Rashidian, ${ }^{1}$ Kaitlyn O'Connor, ${ }^{5}$ Rodolfo Chaparro, ${ }^{5}$ Ronald Seidel, ${ }^{2}$ Maia Mesyngier, ${ }^{2}$ Ross Cheloha, ${ }^{2} J a s o n$ Dearling, ${ }^{1}$ Phaneendra Duddempudi, ${ }^{1}$ Alev Celikgil, ${ }^{1}$ Scott Garforth, ${ }^{2}$ Alan Packard, ${ }^{1}$ Harris Goldstein, ${ }^{2}$ Hidde Ploegh, Steven Almo*. 'Albert Einstein College of Medicine, Bronx, NY, USA; '²Boston Children's Hospital, Boston, MA, USA; ${ }^{3}$ Massachusetts Institute of Technology, Cambridge, MA, USA; ${ }^{4}$ Dana Farber Cancer Institute, Boston, MA, USA; ${ }^{5}$ Cue Biopharma, Cambridge, MA, USA

Background Immunotherapies are highly promising and effective strategies for the treatment of cancer; however, continuing challenges persist, including 1) untargeted global immune modulation, resulting in serious side effects; 2) lack of therapeutics capable of in vivo expansion of tumor-specific $\mathrm{T}$ cells; 3) inability to visualize in vivo tumor-specific $\mathrm{T}$ cell responses; and 4) lack of flexible platforms to rapidly and efficiently explore new therapeutic strategies and immune-escape mechanisms. To address these challenges, we developed a novel class of precision biologics to treat cancer, autoimmune diseases and infectious diseases. We describe a modular platform constructed around an Fc-based covalent pMHC dimer, referred to as synTac (artificial synapse for $\mathrm{T}$ cell activation; also termed Immuno-STATs for Selective Targeting and Alteration of $\mathrm{T}$ cells), which selectively delivers different cargoes, including costimulatory, coinhibitory or cytokine signals and other modalities to primary $\mathrm{T}$ cells of defined specificity. The inherent modularity supports broad applications. Changing the encoded peptide enables targeting of different $\mathrm{T}$ cell specificities to address different diseases, while altering the cargo allows for evaluation of different co-modulatory mechanisms or the delivery of mechanistically informative probes.

Methods Sortase A-mediated enzymatic coupling supported site-specific and stoichiometric installation of positron emission tomography (PET)-active radiolabels on synTacs to visualize the in vivo localization of antigen-specific $T$ cells. The NSG humanized mouse model allowed for the evaluation of synTacs/Immuno-STATs to drive the in vivo antigen-specific expansion of human CD8 T cells. 